\title{
As redes de colaboração no turismo de base comunitária: implicações para a gestão participativa
}

\author{
Collaborative networks in community-based tourism: implications for participatory management
}

\section{Andrés Burgos}

Universidade de Brasília, Centro de Desenvolvimento Sustentável (CDS), Campus Universitário Darcy Ribeiro, Gleba A - 70904-970 Brasília-DF, Brasil, anburgosdelgado@unb.br

\section{Frédéric Mertens}

Universidade de Brasília, Centro de Desenvolvimento Sustentável (CDS), Campus Universitário Darcy Ribeiro, Gleba A - 70904-970 Brasília-DF, Brasil, mertens.br@gmail.com

\section{Resumo}

O turismo de base comunitária (TBC) é o resultado de um processo de colaboração intracomunitário baseado em relações interpessoais, no qual a rede social se apresenta como elemento que tem a capacidade de medir a extensão da colaboração. O presente trabalho discute o TBC assumindo que o processo de gestão da atividade turística supõe envolvimento em relações sociais. Para avaliar empiricamente a implicação do padrão relacional na gestão turística, aplicou-se a análise de redes sociais (ARS) na rede de colaboração do TBC da Prainha do Canto Verde (Ceará, Brasil). O principal objetivo é contribuir com a reflexão teórico-metodológica sobre como os padrões relacionais dentro de uma comunidade podem lançar luz sobre a gestão participativa no turismo local, atendendo à complexidade que caracteriza as relações sociais e o sistema turístico. Os resultados da ARS mostraram que a questão chave para o desenvolvimento de uma gestão do TBC ancorada na equidade participativa é a coexistência das relações entre diferentes atores. As conclusões apontam igualmente para a pertinência da abordagem de redes na análise dos processos e resultados do desenvolvimento turístico comunitário.

Palavras-chave: Turismo de base comunitária, gestão participativa, sustentabilidade, redes sociais, colaboração.

\section{Abstract}

Community-based tourism (CBT) is the result of an intra-community collaborative process, based on interpersonal relationships and in which social networks have the capacity to measure the extent of collaboration. This paper discusses community-based tourism under the assumption that both the concept of community and the process of managing touristic activities imply being involved in social relations. Social network analysis (SNA) was applied to investigate the collaborative network of Prainha do Canto Verde (Ceará, Brazil) in order to empirically assess the implications of relational patterns in tourism management. The main objective is to contribute to theoretical and methodological insights on how relational patterns within a community can shed light on participatory management in local tourism, given the complexity that characterizes social relations and the tourism system. Results of the SNA study showed that the key issue for the development of a CBT management anchored in equity and participation is the coexistence of relations between different actors. The findings also demonstrate the relevance of a network approach in the analysis of processes and results of community-based tourism development.

Keywords: Community-based tourism, participatory management sustainability, social networks, collaboration.

\section{Introdução}

O turismo é uma atividade com potencial para provocar modificações substanciais na qualidade de vida das populações locais, assim como experiências gratificantes ou insatisfatórias nos visitantes, em função do respeito dos seus próprios limites de desenvolvimento econômico e de exploração ambiental e sociocultural (Swarbrooke, 2000; Krippendorf, 2003). A construção e viabilidade de um modelo turístico sustentável pressupõe atenção às questões relacionadas à gestão participativa, mediante a integração das populações locais nos processos de desenvolvimento turístico, de forma que essas deixem de ser apenas espectadoras de ditos processos e tornem-se verdadeiras protagonistas, gestoras e beneficiárias da própria atividade (Hall, 2001; Graci, 2013).

O turismo de base comunitária ( $\mathrm{TBC}$ ) é uma prática turística diferenciada do turismo convencional que visa a promoção da participação de todos os membros da comunidade envolvidos na atividade. Sendo assim, a compreensão e a análise do TBC exigem uma abordagem sistêmica que examine os processos colaborativos entre os diferentes membros da comunidade como um elemento chave do sucesso da atividade. Isso porque o sucesso do TBC está atrelado ao caráter complementário de produtos e serviços e à necessidade de estabelecer parcerias para a gestão dos recursos turísticos.
A visão sistêmica do TBC e a interpretação da participação como o relacionamento existente dentro de tal sistema fazem com que o estudo das redes sociais aplicadas a essa atividade possa oferecer resultados interessantes para a análise da gestão participativa nos destinos e organizações turísticas de base comunitárias (Scott, Baggio \& Cooper, 2008; Erkus-Özturk \& Eraydin, 2010; Burgos, 2014; Burgos \& Mertens, 2015). Desse modo, a análise de redes sociais (ARS) pode contribuir no entendimento das complexas relações necessárias para a operacionalização das atividades e a elaboração do produto turístico.

O objetivo deste trabalho é avaliar a utilidade da abordagem de redes sociais como uma forma para compreender as implicações que a articulação comunitária, através de relações de colaboração, têm para o desenvolvimento de projetos de TBC. Além disso, pretende propiciar subsídios e elementos teórico-metodológicos para entender o funcionamento dos grupos e/ou indivíduos envolvidos num processo colaborativo de TBC e poder, assim, desenredar sua capacidade de ação coletiva.

Partindo dessa perspectiva, a primeira seção do artigo apresenta, sucintamente, o TBC como prática turística que requer relações de colaboração intracomunitárias para gerenciar os serviços e recursos locais. Na sequência, expõem-se alguns elementos que permitem entender o potencial da ARS como marco teóricometodológico para caracterizar os padrões de interação entre os membros de uma comunidade envolvida no TBC e avaliar as 
relações entre estes padrões e a gestão participativa da atividade. A seção seguinte apresenta um estudo de caso no qual a ARS é aplicada para revelar os vínculos entre o padrão estrutural da rede comunitária de colaboração na atividade turística e a gestão participativa do TBC. Por fim, são apresentadas a discussão e as considerações finais do estudo.

\section{Turismo de Base Comunitária: um processo colaborativo}

O TBC surgiu como uma alternativa e processo de resistência ao modelo de turismo convencional (Coriolano, 2009; Irving, 2009). Essa modalidade turística incorpora o estabelecimento de novas formas de trabalho baseadas na colaboração em prol de objetivos comuns. Isso se expressa mediante um planejamento fundamentado em um exercício consciente de corresponsabilidade, de participação local e de governança compartilhada; com o objetivo de, além de dar resposta à demanda dos visitantes, conciliar o desenvolvimento local e a conservação da natureza (Manyara \& Jones, 2007; Lesego, 2010).

Embora não exista uma definição amplamente aceita do TBC, é possível destacar algumas premissas que indicam seu alcance e objetivos. Entre elas: apropriação e empoderamento das comunidades no planejamento, implementação e avaliação dos projetos; escala limitada e conservação dos recursos naturais e/ou culturais; desenvolvimento econômico e social para a comunidade local; e qualidade da experiência do visitante (Hiwasaki, 2006; Irving, 2009). Entretanto, o que verdadeiramente torna singulares as experiências de TBC são a organização e a participação social da comunidade, assim como o predomínio das relações sociais colaborativas nos serviços turísticos implementados (Hall, 2001; Fabrino, Costa \& Nascimento, 2012).

A perspectiva de autogestão das iniciativas que caracterizam o TBC exige permanente interlocução e negociação de compromissos intracomunitários, de modo que a população local assuma o controle pleno no desenvolvimento turístico (Lee, 2013). Os membros da comunidade têm que incorporar estratégias de colaboração para coordenar os elementos que conformam o "pacote turístico", tais como transporte, hospedagem, alimentação e entretenimento, e dar resposta às necessidades dos turistas. Além disso, minimizar as ameaças potenciais do turismo sobre o local, de forma a preservar os atrativos turísticos e alcançar o benefício coletivo para a comunidade, passará pela implementação de alianças mutuamente benéficas que sejam economicamente rentáveis e socialmente aceitáveis para a população local.

A colaboração entre as partes interessadas está sujeita ao reconhecimento de que as questões a resolver são importantes para a comunidade e que atingir benefícios significativos será o resultado de trabalho conjunto. Ao trabalhar coletivamente, os membros da comunidade envolvidos na gestão do TBC podem trocar informações e experiências, aprender uns com os outros, fornecer novas oportunidades e soluções, se adaptar com sucesso a ambientes em constante evolução e canalizar energias para a gestão de bens comuns (Aref, Gill \& Aref, 2010). Além disso, ao envolver o aprendizado coletivo, as relações de colaboração entre os membros da comunidade promovem decisões que gozam de um maior grau de consenso e respaldo social (Araujo \& Bramwell, 1999).
Desse modo, as relações de colaboração intracomunitárias fornecem um quadro base para a resolução de questões organizacionais e operacionais no desenvolvimento do turismo em nível local e podem ser usadas para avançar em visões compartilhadas, nas quais os membros de uma comunidade reconhecem as vantagens potenciais de trabalhar juntos.

\section{O papel das redes sociais na gestão do turismo de base} comunitária

O sucesso do TBC está condicionado a existência de relações de colaboração entre os membros da comunidade envolvidos na atividade. Tais relações devem ser levadas em consideração no âmbito de dois aspectos fundamentais que, juntos, compõem o produto turístico e se apresentam como o principal desafio da proposta: (1) a gestão coletiva das atividades turísticas; (2) a governança compartilhada dos recursos naturais e culturais. Por um lado, a colaboração associada à organização dos serviços pode implicar, entre outras coisas, a melhoria das opções de lazer prestadas e da gestão dos empreendimentos, assim como o acesso ao mercado e a consolidação do destino, fundamental para a sustentabilidade econômica das práticas de TBC (Okazaki, 2008; Bursztyn \& Bartholo, 2012). Por outro lado, as relações de colaboração na gestão responsável dos recursos locais permitiriam que o destino se mantivesse por mais tempo fora da zona de declínio que o modelo do ciclo de vida prevê (Butler, 1980), possibilitando uma experiência dos viajantes satisfatória e uma relação mais favorável ao ambiente em que estão inseridos.

O planejamento turístico comunitário tem que incorporar uma estrutura relacional que fomente a participação de todos os interessados, minimizando os desequilíbrios na rede de interdependências e trabalhando para a integração das partes, por meio de mecanismos que garantam a sustentabilidade do sistema (Landorf, 2009). Desde uma perspectiva relacional, a gestão compartilhada tanto dos serviços turísticos, quanto dos recursos naturais e culturais que constituem os atrativos turísticos, exige que os membros da comunidade estejam dispostos e sejam capazes de colaborar uns com os outros de forma inclusiva e horizontal.

A ARS permite identificar estruturas relacionais às quais se deve atribuir a emergência de propriedades sistêmicas da rede, que ajudarão a discernir melhor tanto a organização da comunidade quanto os seus resultados no desenvolvimento do TBC. A ARS é um enfoque e conjunto de técnicas usadas para estudar o intercâmbio de recursos entre atores, tais como indivíduos, grupos ou organizações (Knoke \& Yang, 2008; Marin \& Wellman, 2011). Centra-se nos padrões de relação entre os atores e examina a disponibilidade e troca de recursos entre eles (Wasserman \& Faust, 1999; Scott, 2000). Assim, a ênfase situa-se nos padrões estruturais das relações, em vez de nos atores isolados, e em como a estrutura das redes pode influir nos processos de gestão (Pretty, 2003; Lauber, Decker \& Knuth, 2008; Scott et al., 2008; Bodin \& Crona, 2009; Presenza \& Cipollina, 2010).

As redes sociais apresentam-se como fatores relevantes no fomento e no fortalecimento tanto da gestão participativa das atividades turísticas, quanto dos processos colaborativos de governança sócio-ambiental (Halme, 2001; Dredge, 2006; Lauber 
et al. 2008; Scott et al., 2008; Erkus-Özturk \& Eraydin, 2010; Mertens, Távora, Fonseca, Grando, Castro \& Demeda, 2011; Rathwell \& Peterson, 2012). O interesse na participação social dos atores envolvidos, mediante a criação de redes sociais de colaboração para o desenvolvimento do TBC, está intimamente ligado à ideia de que os destinos turísticos poderiam obter ganhos mediante a troca de experiências, conhecimentos e outros recursos (Shih, 2006; Baggio, 2011).

Estabelecer relações por meio de redes sociais não só leva a um aumento de possibilidades de ação conjunta e gestão de conflitos, permitindo uma atuação mais coordenada que atenda os diferentes interesses (Bodin \& Crona, 2009), como também pode promover a troca de conhecimento e experiência entre pessoas ou grupos sociais que possuem objetivos compartilhados (Mertens, Saint-Charles, Lucotte \& Mergler, 2008). A articulação em rede entre os atores melhora, também, o desenvolvimento de conhecimentos mediante o intercâmbio e a exposição a novas ideias, facilitando a realização de tarefas complexas (Bramwell \& Lane, 1999). Em termos empresariais, as vantagens das redes são particularmente importantes para a inovação, tal como para construir sistemas de segurança contra a incerteza e clima de negócios desfavoráveis. Mediante redes interorganizacionais, as empresas, bem como os empreendimentos comunitários, têm maior possibilidade de aceder ao financiamento e outros recursos, reduzir assimetrias de informação, assim como possibilitar maior poder de negociação, resultando na melhora das suas capacidades organizativas e competitivas e, por conseguinte, no posicionamento dos empreendimentos colaborativos no mercado (Borgatti \& Foster, 2003; Novelli, Schmitz \& Spencer, 2006).

Do ponto de vista da colaboração na gestão dos recursos turísticos, o discurso do TBC gira em torno à capacidade que a comunidade tem de gerenciar o patrimônio natural e cultural dos destinos, satisfazendo as necessidades dos turistas e promovendo o bemestar comunitário. A esse respeito, cabe enfatizar a abordagem relacional comunitária como uma gestão efetiva dos recursos turísticos no TBC, já que a comunidade pode exercer maior "controle social" e ter a capacidade de enfrentar os problemas que envolve seu uso (Folke, Hahn, Olsson \& Norberg, 2005; Lauber et al., 2008). As redes sociais oportunizam múltiplas perspectivas sobre os objetivos, processos e resultados dos arranjos de governança (Head, 2008). Além disso, os processos de gestão de recursos fomentados pelas redes sociais favorecem a ação coletiva, podendo ser mais efetivos que os instrumentos de comando e controle operados pelas instituições formais (Pretty, 2003). Por fim, as características das redes sociais podem influenciar a capacidade das comunidades para responder às mudanças ambientais, assim como condicionar o sucesso de gestão de recursos naturais (Bodin \& Crona, 2009).

A forma como as redes de colaboração no TBC se organizam e são gerenciadas pode condicionar a inclusão e a participação social e, ainda, comprometer a boa governança para o desenvolvimento sustentável no turismo local (Araujo \& Bramwell, 1999; Pforr, 2006; Erkus-Özturk \& Eraydin, 2010). Desse modo, as características das redes sociais contribuiriam para reforçar a noção de colaboração entre os membros da comunidade, uma vez que tanto a satisfação final do turista no destino quanto a conservação dos recursos não podem ser resolvidas de maneira individual.

As propriedades das redes ajudam a quantificar as características estruturais que, por sua vez, podem evidenciar propriedades importantes para a gestão participativa do TBC, ajudando a entender como os atores estão imersos na rede e como isso pode influir na troca potencial de recursos entre eles. O Quadro 1, fechando a seção, apresenta um leque de opções de medidas e suas implicações para a gestão participativa no TBC. Tais medidas serão examinadas no estudo de caso para ajudar a interpretar o significado que a estrutura relacional de uma rede de colaboração no TBC tem para criar condições favoráveis à sustentabilidade no destino turístico

Quadro 1 - Medidas de redes de colaboração e implicações para a gestão participativa do TBC

\begin{tabular}{|c|c|c|}
\hline Medida de rede & Descrição & Relevância para a gestão participativa do TBC \\
\hline $\begin{array}{l}\text { Diversidade de } \\
\text { grupos de atores }\end{array}$ & $\begin{array}{l}\text { Número de subgrupos de nós definidos por um atributo } \\
\text { comum. }\end{array}$ & $\begin{array}{l}\text { A heterogeneidade e a diversidade de atores envolvidos } \\
\text { num processo colaborativo de TBC podem ampliar a base } \\
\text { de conhecimento coletivo e aumentar a capacidade de } \\
\text { trabalho comunitário. }\end{array}$ \\
\hline $\begin{array}{l}\text { Número de } \\
\text { componentes }\end{array}$ & $\begin{array}{l}\text { Número de subgrupos de atores conectados direta ou } \\
\text { indiretamente por meio de outros indivíduos. Se a rede } \\
\text { contem um ou mais nós isolados, esses atores são } \\
\text { componentes. Se a rede possui diversos componentes, é } \\
\text { considerada fragmentada. }\end{array}$ & $\begin{array}{l}\text { A fragmentação reduz o acesso a contatos entre } \\
\text { subgrupos, necessário para alavancar recursos, ideias, } \\
\text { inovação e informações. Uma gestão participativa do TBC } \\
\text { exigiria a conexão de diferentes grupos envolvidos } \\
\text { mediante laços de colaboração. }\end{array}$ \\
\hline Centro-periferia & $\begin{array}{l}\text { Atores centrais são fortemente ligados entre si, enquanto } \\
\text { atores da periferia apenas estão conectados com os centrais, } \\
\text { mas não com outros periféricos. }\end{array}$ & $\begin{array}{l}\text { Esta característica tem implicações tanto para o acesso, } \\
\text { quanto para a difusão de diversos recursos. Atores centrais } \\
\text { podem agir de duas maneiras: como pontes, trazendo } \\
\text { informações, conhecimentos, percepções e inovações; e } \\
\text { como núcleos de comunicação, disseminando esses } \\
\text { recursos disponíveis. A estrutura centro-periferia pode ter } \\
\text { efeitos também sobre a ação coletiva na rede de } \\
\text { colaboração do TBC, por meio da capacidade de facilitar a } \\
\text { coordenação e a divisão de tarefas. }\end{array}$ \\
\hline
\end{tabular}




\begin{tabular}{|c|c|c|}
\hline Medida de rede & Descrição & Relevância para a gestão participativa do TBC \\
\hline Densidade & $\begin{array}{l}\text { Proporção de relações existentes na rede em relação às } \\
\text { potencialmente presentes, se cada indivíduo possuísse } \\
\text { relações com todos os outros. }\end{array}$ & $\begin{array}{l}\text { Alta densidade: aumento da possibilidade de exposição a } \\
\text { novas ideias e acesso a uma maior quantidade de } \\
\text { informações e recursos; maior potencial de ação coletiva } \\
\text { devido aos níveis de reciprocidade e confiança mútua entre } \\
\text { os atores alcançados ao longo do tempo. Pode trazer } \\
\text { efeitos positivos sobre a capacitação e qualidade dos } \\
\text { serviços turísticos. Porém, pode conduzir à } \\
\text { homogeneização de atitudes e a menor especialização de } \\
\text { conhecimento, devido à "sobrecarga" ou saturação da } \\
\text { rede com as mesmas informações. Isso pode levar a uma } \\
\text { menor eficiência da rede de TBC na utilização de recursos } \\
\text { e à redução da capacidade de adaptação à mudanças. } \\
\text { Baixa densidade: fracas relações de socialização entre os } \\
\text { membros da rede e redução dos processos colaborativos } \\
\text { da comunidade como um todo para o desenvolvimento da } \\
\text { atividade turística. }\end{array}$ \\
\hline $\begin{array}{l}\text { Distribuição do } \\
\text { numero de } \\
\text { relações entre } \\
\text { indivíduos }\end{array}$ & $\begin{array}{l}\text { Frequência relativa de indivíduos com um número de } \\
\text { relações igual a } 1,2,3 \text {, etc. }\end{array}$ & $\begin{array}{l}\text { Distribuição homogênea: descentralização das relações de } \\
\text { poder (horizontalidade). Todos os atores ocupam posições } \\
\text { igualmente centrais e colaboram por igual no TBC. } \\
\text { Distribuição heterogênea: colaborações centralizadas } \\
\text { entorno a um ou vários indivíduos da comunidade que } \\
\text { controlam as interações e direcionam a gestão turística. }\end{array}$ \\
\hline $\begin{array}{l}\text { Número médio } \\
\text { de relações } \\
\text { internas aos } \\
\text { grupos e entre } \\
\text { grupos }\end{array}$ & $\begin{array}{l}\text { Distribuição de relações entre indivíduos do mesmo grupo } \\
\text { social (bonding) e entre indivíduos de diferentes grupos } \\
\text { sociais (bridging). }\end{array}$ & $\begin{array}{l}\text { Os vínculos tipo bonding favorecem a troca de informações } \\
\text { e, potencialmente, a promoção da ação coletiva e gestão } \\
\text { de conflitos internos. Já os laços bridging facilitam o acesso } \\
\text { a novas ideias e à mobilização de recursos que não estão } \\
\text { disponíveis dentro de um mesmo grupo. No TBC, um } \\
\text { equilíbrio entre as relações de colaboração internas aos } \\
\text { diferentes grupos sociais da comunidade e as relações } \\
\text { entre membros da comunidade que pertencem a } \\
\text { diferentes grupos pode ser importante para o processo de } \\
\text { gestão participativa, uma vez que diferentes atores } \\
\text { precisam trabalhar de maneira integrada para o } \\
\text { desenvolvimento da atividade. }\end{array}$ \\
\hline
\end{tabular}

Fonte: elaboração própria a partir de Borgatti \& Jones (1998); Woolcock \& Narayan, 2000; Hanneman (2005); Bodin, Crona \& Ernstson (2006); Bodin \& Crona $(2008,2009)$.

\section{Metodologia}

\subsection{Estudo de caso}

A comunidade da Prainha do Canto Verde localiza-se no litoral leste do Estado do Ceará, a 126 km da capital Fortaleza, e em área de elevado valor econômico, entre alguns dos principais destinos turísticos do litoral cearense. A vila é composta por uma população de aproximadamente 1.000 habitantes, que têm na pesca artesanal sua atividade principal. A localização da comunidade é mostrada na Figura 1.

Figura 1 - Mapa de localização da comunidade da Prainha do Canto Verde

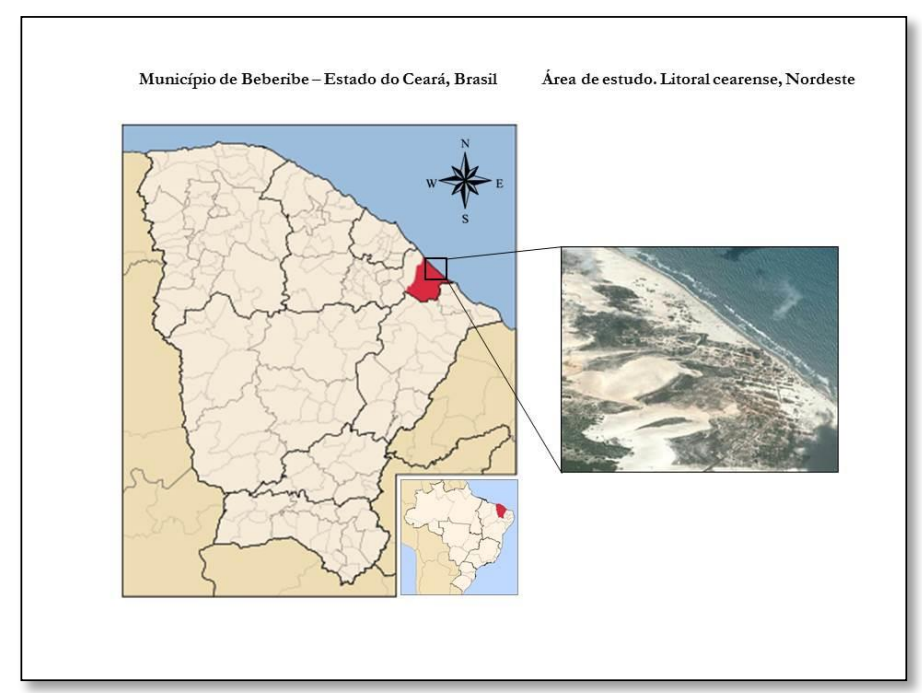

Fonte: Elaboração dos autores a partir de Google Earth. 
O turismo na comunidade começou informalmente no início da década de 90 como uma alternativa para minimizar os impactos negativos da especulação imobiliária sobre o frágil ecossistema litorâneo e da falta de oportunidades de emprego sobre a situação socioeconômica da população local. Esse movimento de resistência na comunidade culminou, em 1997, na elaboração do projeto de TBC da Prainha do Canto Verde. O projeto visa o controle, por parte da comunidade, sobre as ações relacionadas com o turismo, desde o planejamento até o desenvolvimento e gestão das atividades, mediante o envolvimento participativo, descentralizado e associativo, com o objetivo de garantir a melhoria das condições de vida da população local (Schärer, 2003).

A atividade turística da comunidade conta com uma estrutura que engloba restaurantes, pequenas pousadas, casas e quartos de aluguel. Além disso, existem alguns bares e lanchonetes. Quanto às atividades oferecidas aos turistas, estas incluem essencialmente trilhas ecológicas, excursões e passeios de buggy, barco e catamarã. O modelo de turismo da Prainha do Canto Verde é responsável por um fluxo de turistas representado, em sua grande parte, por pessoas procedentes do estado do Ceará e, principalmente, da capital Fortaleza. Normalmente, esses turistas procuram este destino motivados pelas belezas naturais, a tranquilidade e o convívio com a população local. Desde que a proposta começou, o turismo desenvolvido na Prainha do Canto Verde tornou-se a experiência de TBC brasileira de maior sucesso, reconhecida tanto nacional como internacionalmente pela sua forma de organização social e referência para outras comunidades do litoral brasileiro (Bartholo, Sansolo \& Buszrtyn, 2009).

\subsection{Colaboração intracomunitária}

A análise da colaboração no TBC da Prainha do Canto Verde requer a definição da rede social em termos de nós e laços. Na ARS, normalmente, nós representam pessoas ou organizações e laços simbolizam relações entre os nós, tais como padrões de comunicação, colaboração ou intercâmbio de recursos (Wasserman \& Faust, 1999; Scott, 2000; Borgatti, Mehra, Brass \& Labianca, 2009). Neste estudo, os nós representam os membros da comunidade e os laços sociais representam as relações de colaboração entre os membros em matéria de gestão do TBC.

A pesquisa de campo foi realizada durante os meses de abril e maio de 2013 e organizada em duas etapas metodológicas: (1) identificação da fronteira e dos atores da rede e; (2) caracterização dos atores e das relações de colaboração. Para definir a fronteira da comunidade e identificar os membros da rede foram considerados os indivíduos maiores de idade (18 anos) que se autorreconheceram como membros da comunidade e que declararam envolvimento com o TBC. O último critério envolve tanto a participação em alguns aspectos específicos que compõem a experiência turística como, por exemplo, a administração de serviços de hospedagem, o cuidado da alimentação e/ou o fornecimento de serviços recreativos; quanto o envolvimento em diversos arranjos para a gestão e o aproveitamento sustentável dos recursos locais. A identificação dos atores se realizou por meio da elaboração de um censo da comunidade, visitando cada casa e confeccionando um mapa no qual foi atribuído um identificador (ID) correlativo a cada casa. O censo contabilizou um total de 780 pessoas, das quais 413 correspondem a indivíduos maiores de idade (200 homens e 213 mulheres). Quando nenhum morador estava presente no domicílio no momento da visita, uma nova tentativa de contato foi realizada em horário e/ou dia diferente. Nos casos em que a ausência foi repetida após várias visitas, e em função do tempo disponível para a realização do censo, essa casa foi descartada do universo amostral. Ao todo, de um total de 222 casas identificadas, apenas 7 ficaram fora do censo e, portanto, seus moradores não foram contemplados nas análises deste estudo. $\mathrm{Na}$ mesma hora da realização do censo, foram também identificados os moradores maiores de idade que afirmaram trabalhar com o turismo na Prainha do Canto Verde (124 indivíduos, 56 homens e 68 mulheres) e que formam a população amostral de interesse para a análise da rede de colaboração no TBC.

Em seguida, uma vez identificadas as residências e as pessoas que moravam em cada casa, um questionário foi aplicado a aquelas pessoas envolvidas no TBC para a obtenção dos dados atributivos e relacionais. Todos os membros da comunidade que declararam envolvimento com o TBC foram entrevistados. Com o objetivo de identificar as relação de colaboração, o questionário solicitava que a pessoa entrevistada citasse, livremente, nomes de outros membros da comunidade com que ela forma alianças, se relaciona e/ou estabelece parcerias para desenvolver a atividade (ou atividades) turística citada previamente. A caracterização dos membros da rede se baseou em variáveis demográficas e socioculturais identificadas a partir do contexto local e da revisão da literatura que podem condicionar as relações de colaboração e os padrões de uso de recursos (Ramirez-Sanchez, 2011; BarnesMauthe, Arita, Allen, Gray \& Leung, 2013): sexo, idade, origem, escolaridade, envolvimento em associações locais, atividade ocupacional principal, ocupação relacionada com o turismo e tempo trabalhando com o turismo. Esses dados atributivos serviram para caracterizar a população amostral e estabelecer a diversidade de grupos presentes.

Para aproveitar ao máximo as informações obtidas na pesquisa de campo, nesse estudo se adotou a simetria máxima de relações de colaboração. Isto significa que não foi necessária a citação recíproca nas entrevistas para considerar a existência de relação de colaboração. Os dados coletados foram analisados com o programa de tratamento de dados relacionais UCINET 6 (Borgatti, Everett \& Freeman, 2002) e exportados para o software Netdraw (Borgatti, 2002) para visualizar a estrutura da rede de colaboração.

\section{Resultados e discussão}

A rede de colaboração do TBC na Prainha do Canto Verde está formada por 124 indivíduos e apresenta um número médio de conexões de 6,79. O tamanho da rede corresponde a $30 \%$ da população adulta da comunidade. Os resultados mostram que $42,5 \%$ do total das famílias identificadas no censo têm algum membro trabalhando no turismo. Esses dados são condizentes com outros estudos que apontam à necessidade de desmitificar a ideia de coletividade no TBC, uma vez que o turismo comunitário envolve, em geral, apenas uma parcela dos membros da comunidade (Blackstock, 2005; Ruiz, Hernández, Coca, Cantero \& Campo, 2008).

Levando em consideração as variáveis demográficas e socioculturais que podem condicionar as relações de colaboração (listadas na seção anterior), a rede de estudo apresenta uma diversidade de grupos de atores. Um exemplo desta diversidade é ilustrado na Figura 2, onde os atores estão identificados de acordo com a ocupação desenvolvida no turismo. 
Figura 2 - Rede de colaboração de turismo comunitário na Prainha do Canto Verde $(n=124)$ por ocupação desenvolvida no turismo. Quadrados representam homens e círculos representam mulheres

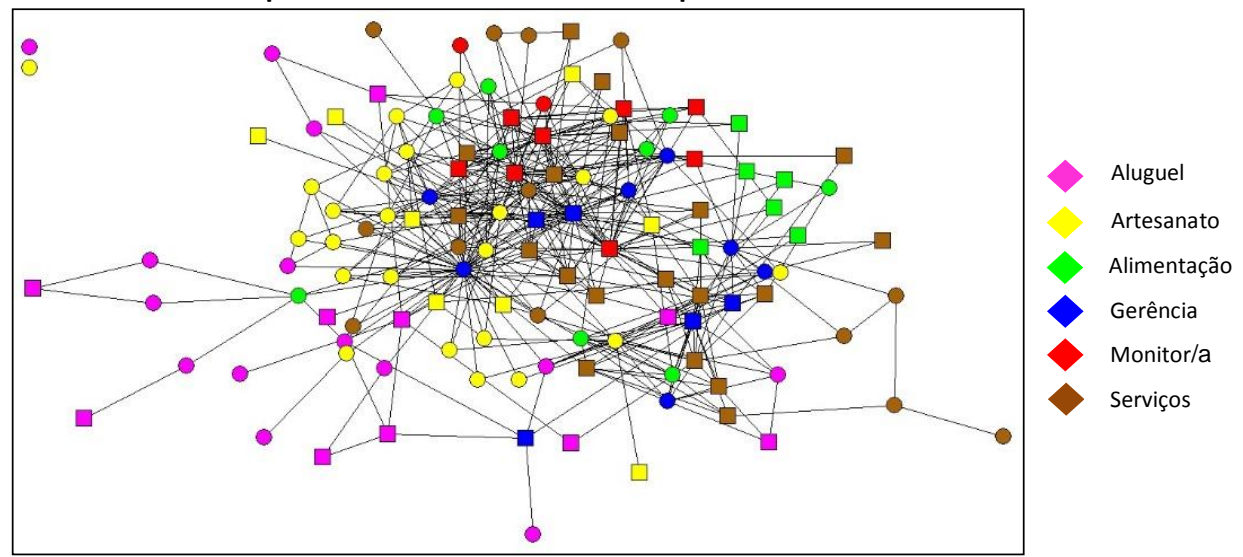

Fonte: Elaboração dos autores.

É comumente admitido que uma diversidade de grupos dentro de um sistema ajuda a construir a robustez (Webb \& Bodin, 2008; Bodin \& Crona, 2009). Essa diversificação aumenta as chances de que a rede de colaboração do TBC permaneça estável diante de eventuais ameaças, já que se incrementa a probabilidade de os grupos envolvidos na gestão do TBC ofereçam respostas diferentes para perturbações. Ainda, a variedade de grupos diminui o risco de que a rede de colaboração alcance a homogeneização de conhecimentos e experiências, aumentando assim a capacidade dos atores para se adaptar às condições de mudança (Bodin e Norberg, 2005). Desta forma, por exemplo, numa situação na qual cada artesão estabelece parcerias com mais de uma gerência de pousada, se uma desaparecer, as pessoas que realizam artesanato podem continuar trabalhando com outras pousadas, tendo mais opções de garantir a continuidade da ocupação e, consequentemente, a própria renda. 0 mesmo pode acontecer com as pousadas que dispõem de diversas opções de fornecimento de alimentos ou serviços, assim como com as pessoas que utilizam seus contatos diversificados na rede para aumentar as chances de alugar sua casa ou algum quarto aos turistas. Assim, a diversidade de grupos envolvidos na gestão do TBC favorece a capacidade de adaptação da rede, bem como sua robustez, mas também a realização das múltiplas tarefas necessárias para satisfazer a experiência recreativa dos visitantes.

Outra aproximação simples e comumente utilizada na ARS baseiase na observação da estrutura global e na identificação de subestruturas que podem ser caracterizadas pelo número de componentes, ou seja, de partes da rede que estão internamente conectadas, mas desconectadas entre elas. Esta caracterização permite detectar vulnerabilidades ou pontos fracos como linhas de separação na estrutura geral de conexão da rede. A rede de colaboração da Prainha do Canto Verde possui três componentes (Figura 2). O componente principal está formado pela maioria dos membros da rede, densamente conectados entre si $(n=122)$ e que, portanto, podem trocar experiências, informações e recursos para a gestão do TBC. Os outros dois componentes da rede são dois atores isolados. Isso porque, embora esses indivíduos se autorreconheceram como partes envolvidas na gestão do TBC, não citaram parceiro de colaboração algum, nem foram indicados por outros. Os atores isolados correspondem a duas mulheres da comunidade potencialmente excluídas dos processos colaborativos e de aprendizagem na gestão do TBC. A elevada proporção de membros conectados mediante relações de colaboração em um só componente $(98,3 \%)$ define a capacidade que a rede possui para o controle social da atividade e a ação conjunta na gestão dos serviços e recursos turísticos, especialmente quando, como visto, a rede envolve relações com diversos grupos de atores. Além disso, favorece que as decisões a respeito do desenvolvimento do TBC sejam mais coletivas.

Em configurações do tipo centro-periferia, atores no centro estão densamente conectados entre si, enquanto os atores agrupados na periferia apresentam maior densidade de laços com os atores do centro do que com seus pares periféricos. Isso significa que a rede só possui um subgrupo fortemente unido, no centro. Uma estrutura centro-periferia é um meio para medir o quão distante do centro está cada ator da rede e também pode ser caracterizada pela ausência de subgrupos relativamente coesos (clusters) (Borgatti \& Everett, 1999). A análise centro-periferia da rede de colaboração da Prainha do Canto Verde confirma a configuração da rede nesse padrão estrutural. $O$ resultado do procedimento analítico é ilustrado na Figura 3.

Figura 3 - Estrutura centro-periferia da rede de colaboração de turismo comunitário na Prainha do Canto Verde

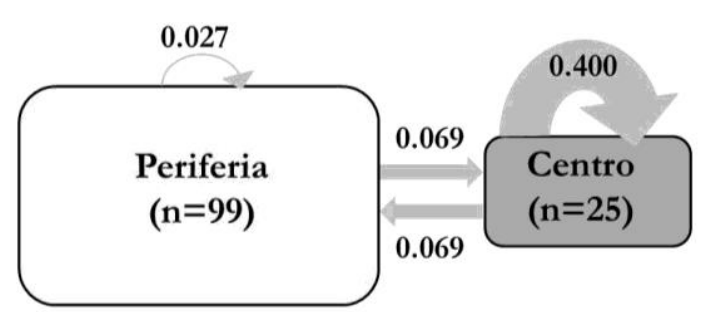

Fonte: Elaboração dos autores.

A análise mostra a constituição de um grupo central formado por 25 membros da rede (16 homens e 9 mulheres) sumamente coesos, ficando os demais na periferia. A densidade do centro é 0,40 , o que significa que os atores da zona central mantêm entre si o $40 \%$ de todas as colaborações possíveis entre os membros da rede. Esse conglomerado de membros da rede de colaboração constitui o núcleo envolta do qual se organiza a atividade do TBC. Os membros da rede assignados à zona da periferia encontram-se 
pouco coesos, ainda que essa coesão seja maior com os membros da zona central (densidade: 0,069) do que com seus pares (densidade: 0,027).

As redes centro-periferia facilitam a coordenação da ação coletiva e processos de governança. Em comparação com as redes formadas por múltiplos centros ou grupos distinguíveis, as estruturas centro-periferia apresentam apenas um "centro de poder", fato que pode reduzir o risco de conflitos que venham eventualmente a dificultar os processos colaborativos (Johnson, Boster \& Palinkas, 2003). Na rede de estudo, essa característica estrutural tem implicações, por exemplo, para o acesso e a difusão de informação e conhecimento diversificado. Capacidades e conhecimentos diversificados podem ajudar à comunidade a desenvolver estratégias de organização de atividades conjuntas e/ou projetos comuns, assim como a estabelecer regras ou tomar decisões melhor justificadas, atendendo as necessidades de cada caso. Adicionalmente, a estrutura centro-periferia da rede do TBC afeta positivamente à capacidade de mobilização que a rede possa precisar em momentos críticos de gestão das atividades, já que a alta conectividade entre os atores centrais facilita a tomada de decisão e permite que as informações possam também alcançar rapidamente o conjunto de atores da periferia.

A densidade da rede de colaboração da Prainha do Canto Verde é $5,52 \%$. Porém, esse indicador não oferece muitas explicações analíticas uma vez que a medida varia segundo o tipo e a força da relação considerada, assim como dependendo do tamanho da rede estudada. Uma alternativa para avaliar a densidade global de uma rede e poder comparar redes de tamanhos diferentes é o numero médio de relações por individuo (ou centralidade de grau). Esta medida representa o envolvimento dos membros da rede na colaboração para a gestão do TBC. Os valores de centralidade dos atores mais conectados e a média de centralidade da rede estudada são apresentados na Tabela 1.

\section{Tabela 1 - Resultados da centralidade na rede de colaboração de estudo para os cinco atores mais centrais}

\begin{tabular}{|c|c|c|c|}
\hline ID & Sexo & Ocupação no TBC & $\begin{array}{c}\text { Centralidade de grau } \\
\text { (Degree) }\end{array}$ \\
\hline P12702 & F & Gerente de pousada & 46,00 \\
\hline P12701 & M & Gerente de pousada & 34,00 \\
\hline P01303 & M & Monitor & 30,00 \\
\hline P18601 & M & Monitor & 26,00 \\
\hline P09904 & F & Artesanato & 24,00 \\
\hline Média de centralidade de todos os nós & 6,70 \\
\hline
\end{tabular}

Fonte: Elaboração dos autores. O ID corresponde ao identificador atribuído a cada ator para garantir seu anonimato.

Os indivíduos mais conectados possuem valores de conectividade que diferem bastante da média de toda a rede, evidenciando seu poder potencial enquanto a capacidade de acessar informação e exercer influência direta sobre outros. Embora sejam poucos, a maneira com que os membros da comunidade que ocupam essas posições favoráveis utilizam seu poder terá um impacto positivo ou negativo sobre os resultados da gestão do TBC.

A distribuição de conectividades entre indivíduos é outra propriedade importante da rede, que tem implicações para a geração e transferência de conhecimentos relevantes para a gestão de recursos no TBC. Esta propriedade está relacionada com a dispersão das relações. A frequência de distribuição das relações de colaboração é mostrada na Figura 4.

\section{Figura 4 - Distribuição de frequência (\%) do número de relações de colaboração na rede de colaboração da Prainha do Canto Verde}

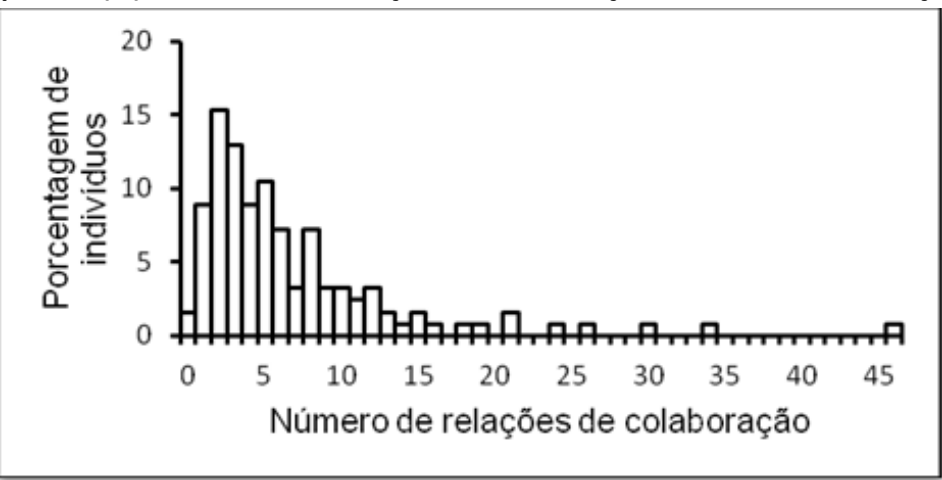

Fonte: Elaboração dos autores.

A rede de colaboração da Prainha oferece uma distribuição heterogênea das colaborações. Isso revela que existe uma alta dispersão das relações, com poucos indivíduos altamente conectados e muitos membros da rede com poucas conexões ( $58 \%$ dos indivíduos têm de 0 a 5 relações de colaboração). Um alto grau de centralidade pode ser uma vantagem em momentos iniciais de um processo e em tempos de mudança, pois permite assegurar coordenação rápida e eficaz entre as diversas partes interessadas.
A diversidade de grupos de atores da rede de colaboração da Prainha do Canto Verde, definidos de acordo com variáveis atributivas escolhidas do contexto local, é a base para avaliar as relações entre indivíduos do mesmo grupo social (bonding) e entre indivíduos de diferentes grupos sociais (bridging). O número médio de relações internas aos grupos e entre grupos revela que o padrão das conexões, segundo cada variável demográfica e sociocultural considerada, é heterogêneo. A Tabela 2 representa a distribuição das relações de colaboração dentro e entre grupos de acordo com a ocupação desenvolvida no turismo. 
Tabela 2 - Distribuição das relações de colaboração na comunidade entre grupos classificados pela ocupação relacionada com o turismo

\begin{tabular}{|c|c|c|c|c|c|c|c|c|c|}
\hline \multirow[t]{2}{*}{ Grupos } & \multirow[t]{2}{*}{$\mathbf{n}^{1}$} & \multicolumn{2}{|c|}{$\mathbf{C T}^{2}$} & \multicolumn{2}{|c|}{$\begin{array}{c}\mathrm{Cl}^{3} \\
\text { Bonding }\end{array}$} & \multicolumn{2}{|c|}{$\begin{array}{c}\mathrm{CE}^{4} \\
\text { Bridging }\end{array}$} & \multirow[t]{2}{*}{$\% \mathrm{Cl} / \mathrm{CT}^{5}$} & \multirow[t]{2}{*}{$\% \mathrm{CE} / \mathrm{CT}^{6}$} \\
\hline & & Soma & Média & Soma & Média & Soma & Média & & \\
\hline Artesanato & 31 & 181 & 21,5 & 94 & 34,3 & 87 & 15,3 & 51 & 49 \\
\hline Alimentação & 15 & 74 & 8,8 & 12 & 4,4 & 62 & 10,9 & 16 & 84 \\
\hline Monitor/a & 10 & 121 & 14,4 & 44 & 16,0 & 77 & 13,6 & 37 & 63 \\
\hline Serviços & 30 & 170 & 20,2 & 50 & 18,3 & 120 & 21,1 & 29 & 71 \\
\hline Total & 124 & 842 & 100 & 274 & 100 & 568 & 100 & & \\
\hline
\end{tabular}

1. n: Número de atores de cada grupo. 2. CT: Colaboração total: Soma das relações de colaboração dos atores do grupo (soma) e número médio de relações de colaboração dos atores do grupo (média). 3. Cl: Colaboração interna: Soma das relações de colaboração entre os atores de um mesmo grupo (soma) e número médio de relações de colaboração entre os atores de um mesmo grupo (média). 4. CE: Colaboração externa: Soma das relações de colaboração dos atores do grupo com os atores de outros grupos (soma) e número médio de relações de colaboração dos atores do grupo com atores de outros grupos (média). 5. \%Cl/CT: Porcentagem de relações de colaboração com o mesmo grupo em relação ao número total de relações de colaboração. 6. \%CE/CT: Porcentagem de relações de colaboração com outros grupos em relação ao número total de relações de colaboração.

Fonte: Elaboração dos autores.

No exemplo mostrado, o padrão relacional sugere que, embora existam diferenças na intensidade e no fluxo da colaboração entre e dentro dos grupos analisados, todos colaboram com todos. Ainda assim, o grupo formado pelas pessoas com responsabilidade na gerência das pousadas se apresenta como grupo central. Esse fato evidencia certa dependência da colaboração turística criada em torno das pousadas, assim como a fragilidade da rede em caso de abandono da atividade por estes membros. Contudo, a colaboração amplamente distribuída entre os diferentes atores turísticos comunitários indica os benefícios que a atuação em uma rede colaborativa, como agentes complementares e não competidores, pode trazer para todas as partes interessadas. As relações de colaboração diversificadas no desenvolvimento do TBC não só permitem a troca de produtos e serviços entre as partes, senão também de conhecimentos e pautas de comportamento envolta de questões chave para o desenvolvimento da atividade. A maior intensidade de colaboração não está sempre associada às relações entre membros da comunidade que compartilham as mesmas características pessoais (homofília). A inexistência de padrões de relações claramente diferenciados entre e dentro dos grupos e a tendência para a interação entre membros não socialmente equivalentes da rede (heterofilia) facilita que os diversos grupos se aproveitem dos benefícios da diversidade e diminui a probabilidade de efeitos redundantes e predeterminados. Contudo, embora a existência de contatos diversificados possa provocar a produção de sobreposições e conflitos entre os membros, os vínculos externos entre diferentes grupos aumentam o potencial de benefício mútuo mediante a complementaridade e a variedade de funções e contatos, fomentando o encontro de novas formas de informação e inovação.

As redes sociais propícias para processos de controle e gestão participativa demandam um equilíbrio entre, por um lado, uma coesão estrutural global e, por outro, a presença de grupos horizontalmente conectados. É precisamente mediante a incorporação na prática das noções de inclusão, horizontalidade e redes de colaboração que é possível aproximar-se do ideal de desenvolvimento sustentável no âmbito do turismo (Dredge, 2006; Erkus-Özturk \& Eraydin, 2010). Por isso, as redes sociais apresentam-se como um mecanismo útil para compreender a função que esses elementos desempenham no processo de desenvolvimento turístico comunitário.

\section{Considerações finais}

O TBC acolhe a participação social como compromisso central do planejamento e gestão da atividade. Quando diferentes membros de uma comunidade que exerce $\mathrm{O}$ TBC trabalham juntos, mediante diversos processos e acordos relacionais, a estrutura que emerge desta inter-relação se traduz na existência de uma rede social. Diversos interesses e graus de participação dos membros envolvidos no TBC podem condicionar a articulação comunitária para o desenvolvimento dos projetos turísticos. Igualmente, diferentes tipologias de participação demandam diferentes tipos de relação e, portanto, podem resultar em configurações de redes sociais específicas, assumindo diversos arquétipos. Embora em graus diferentes em função dos casos, espera-se que modelos de participação associados ao TBC assumam estruturas solidárias e colaborativas baseadas na horizontalidade e inclusão de participação entre seus membros, devido ao forte compromisso social que fundamenta o TBC. Isso se evidencia no estudo de caso da Prainha do Canto Verde mediante a aplicação de algumas medidas de ARS que permitem avaliar o grau de envolvimento individual e de diferentes grupos da comunidade no processo colaborativo do TBC.

As redes são criadas de maneiras diferentes e isso pode ter implicações para a capacidade que os membros da comunidade têm de lidar com os desafios da gestão da atividade turística. Cada padrão estrutural de uma rede corresponde à extensão de poder dos participantes em determinar o resultado final na ação do grupo ou comunidade sobre o desenvolvimento do turismo. Dessa forma, a ARS oferece a possibilidade de examinar os impactos sobre o TBC derivados das relações sociais dos membros da comunidade, ajudando a entender como os atores estão imersos na rede e como isso pode influir na troca potencial de recursos 
entre eles. Por conseguinte, a análise da estrutura relacional da rede de colaboração de comunidades envolvidas no TBC ajuda a identificar se a participação social na gestão da atividade representa um condicionante retórico ou real do desenvolvimento do turismo comunitário e, consequentemente, do ideário de sustentabilidade que diferencia esta prática daquelas próprias do turismo convencional.

Como este estudo mostra, os membros da comunidade que trabalham no TBC têm o poder de gerar um efeito sobre a atividade turística, ou seja, a capacidade de intervir e influenciar no seu desenvolvimento mediante seu poder de atuação e através das relações de colaboração que estabelecem entre eles. Entender a estrutura e dinâmica da rede de colaboração de estudo pode ser aproveitado pela própria comunidade para melhorar ou fortalecer sua organização social, e com isso, promover seu desenvolvimento. Da mesma forma, poderia auxiliar a outras comunidades que buscam a implementação de um turismo que viabilize seus respectivos modos de vida por meio da colaboração e de princípios solidários. Contudo, mais pesquisas poderiam tentar examinar que balanço ou equilíbrio entre as relações de colaboração e quais condições oferecem mais e melhores benefícios para o desenvolvimento do TBC em função dos objetivos perseguidos pela atividade. Futuros trabalhos empíricos, baseados na abordagem de redes e aplicados em diferentes contextos turísticos, podem também incorporar facilmente mais informações sobre os nós e os processos relacionais para revelar as dinâmicas colaborativas que acontecem entre os atores turísticos de um determinado território. Por exemplo, atributos dos nós podem ser utilizados para representar a intensidade de uma relação, enquanto os laços sociais podem ser ponderados em função de atributos, assim como caracterizados pela natureza ou tipologia da relação estabelecida. Finalmente, e embora o estudo proposto não vise comparar redes sociais diferentes dentro do contexto do TBC, este trabalho abre a possibilidade de realizar estudos comparativos que tratem, por exemplo, de estabelecer vínculos entre determinados modelos relacionais e os processos de gestão e desenvolvimento turístico das experiências. Além disso, também permitiria desenvolver estudos longitudinais que analisem a evolução e transformação da mesma rede social de TBC ao logo do tempo, contribuindo, assim, para a reflexão e a avaliação das dinâmicas colaborativas locais.

\section{Referências}

Araujo, L. M., \& Bramwell, B. (1999). Stakeholder Assessment and Collaborative Tourism Planning: The Case of Brazil's Costa Dourada Project. Journal of Sustainable Tourism, 7(3-4), 356-378.

Aref, F., Gill, S. S., \& Aref, F. (2010). Tourism Development in Local Communities: As a Community Development Approach. Journal of American Science, 6(2), 155-161.

Baggio, R. (2011). Collaboration and cooperation in a tourism destination: a network science approach. Current Issues in Tourism, 14 (2), 183-189.

Barnes-Mauthe, M., Arita, S., Allen, S. D., Gray, S. A., \& Leung, P. (2013). The influence of ethnic diversity on social network structure in a commonpool resource system: implications for collaborative management. Ecology and Society, 18(1): 23.

Bartholo, R. S.; Sansolo, D. G.; Buszrtyn, I. (2009). Turismo de base comunitária: diversidade de olhares e experiências brasileiras. Rio de Janeiro: Letra e Imagem.

Blackstock, K. (2005). A critical look at community based tourism. Community Development Journal, 40(1), 39-49.
Bodin, Ö., \& Norberg, J. (2005). Information network topologies for enhanced local adaptive management. Environmental Management, 35, 175-193.

Bodin, Ö., \& Crona, B. I. (2008). Management of Natural Resources at the Community Level: Exploring the Role of Social Capital and Leadership in a Rural Fishing Community. World Development, 36(12), 2763-2779.

Bodin, Ö., \& Crona, B. I. (2009). The role of social networks in natural resource governance. What relational patterns make a difference? Global Environmental Change, 19, 366-374.

Bodin, Ö., Crona, B. I., \& Ernstson, H. 2006. Social Networks in Natural Resource Management: What is There to Learn from a Structural Perspective? Ecology and Society, 11(2), r2.

Borgatti, S. P., \& Jones, C. (1998). Network Measures of Social Capital. Connections, 21(2), 1-36.

Borgatti, S. P., \& Everett, M. G. (1999). Models of Core/Periphery Structures. Social Networks, 21, 375-395.

Borgatti, S. P. (2002). NetDraw: Graph visualization software. Harvard: Analytic Technologies.

Borgatti, S. P., Everett, M. G., \& Freeman, L. C. (2002). UCINet 6 for Windows: software for social network analysis. Harvard, MA: Analytic Technologies.

Borgatti, S. P., \& Foster, P. C. (2003). The network paradigm in organizational research: A review and typology. Journal of Management, 29, 991-1013.

Borgatti, S., Mehra, A., Brass, D., \& Labianca, G. (2009). Network analysis in the social sciences. Science, 323, 892-896.

Bramwell, B., \& Lane, B. (1999). Collaboration and Partnerships for Sustainable Tourism. Journal of Sustainable Tourism, 7, 179-181.

Burgos, A. (2014). El papel de las redes sociales en el desarrollo de prácticas turísticas sostenibles: algunas reflexiones teóricas. Turismo em Análise, 25(1), 75-101.

Burgos, A., \& Mertens, F. (2015). A perspectiva relacional na gestão do turismo de base comunitária. Caderno Virtual de Turismo, 15(1), 81-98.

Bursztyn, I., \& Bartholo, R. (2012). O processo de comercialização do turismo de base comunitária no Brasil: desafios, potencialidades e perspectivas. Sustentabilidade em Debate, 3(1), 97-116.

Butler, R. (1980). The concept of a tourist area life cycle of evolution: implications for the management of resources. Canadian Geographer, 24(1), 5-12.

Coriolano, L. N. (2009). Arranjos produtivos Locais do Turismo Comunitário: atores e cenários em mudanças. Fortaleza: UECE.

Dredge, D. (2006). Networks, Conflict and Collaborative Communities. Journal of Sustainable Tourism, 14(6), 562-581.

Erkus-Özturk., \& Eraydin, A. (2010). Environmental governance for sustainable tourism development: Collaborative networks and organization building in the Antalya tourism region. Tourism management, 31, 113-124.

Fabrino, N. H., Costa, H. A., \& Nascimento, E. P. (2012). Turismo de Base Comunitária (TBC): elementos chaves para aferir seu desempenho na perspectiva da sustentabilidade. Revista Brasileira de Ecoturismo, 5(3): 546-559.

Folke, C., Hahn, T., Olsson, P., \& Norberg, J. (2005). Adaptive governance of social-ecological systems. Annual Review of Environment and Resources, 30, 441-473.

Graci, S. (2013). Collaboration and Partnership Development for Sustainable Tourism. Tourism Geographies, 15(1), 25-42.

Hall, M. C. (2001). Planejamento turístico: políticas, processos e relacionamentos. São Paulo: Contexto.

Halme, M. (2001). Learning for sustainable development in tourism networks. Business strategy and the Environment, 10, 100-114.

Hanneman, R. A. (2005). Introduction to social network methods. Riverside, CA: University of California.

Head, B. W. (2008). Assessing network-based collaborations. Public Management Review, 10(6), 733-749.

Hiwasaki, L. (2006). Community-based tourism: A pathway to sustainability for Japan`s protected areas. Society and Natural Resources, 19, 133-143.

Irving, M. A. (2009). Reinventando a reflexão sobre turismo de base comunitária: inovar é possível? In R. Bartholo, D. G. Sansolo, \& I. Bursztyn 
(orgs.), Turismo de base comunitária: Diversidade de olhares e experiências brasileiras (pp. 108-119). Rio de Janeiro: Letra e Imagem.

Johnson, J.C., Boster, J.S., \& Palinkas, L.A. (2003). Social roles and the evolution of networks in extreme and isolated environments. Journal of Mathematical Sociology, 27, 89-121.

Knoke, D., \& Yang, S. (2008). Social Network Analysis. Califórnia: SAGE.

Krippendorf, J. (2003). Sociologia do Turismo: por uma nova compreensão do lazer e das viagens. São Paulo: Aleph.

Landorf, C. (2009). Managing for sustainable tourism a review of six cultural world heritage sites. Journal of Sustainable Tourism, 17(1), 53-70.

Lauber, T. B., Decker, D. J., \& Knuth, B. A. (2008). Social Networks and Community-Based Natural Resource Management. Environmental Management, 42, 677-687.

Lee, T. S. (2013). Influence analysis of community resident support for sustainable tourism development. Tourism Management, 34, 37-46.

Lesego, S. S. (2010). Community-based tourism ventures, benefits and challenges: Khama Rhino Sanctuary Trust, Central District, Botswana. Tourism Management, 31, 136-146.

Manyara, G., \& Jones, E. (2007). Community-based tourism enterprises development in Kenya: An exploration of their potential as avenues of poverty reduction. Journal of Sustainable Tourism, 15(6), 628-644.

Marin, A., \& Wellman, B. (2011). Social network analysis: an introduction. In P. J. Carrington, \& J. Scott (eds.), The Sage Handbook of Social Network Analysis (pp. 11-25). London: SAGE.

Mertens, F., Saint-Charles, J., Lucotte, M., \& Mergler, D. (2008). Emergence and Robustness of a Community Discussion Network on Mercury Contamination and Health in the Brazilian Amazon. Health Education \& Behavior, 35(4), 509-521.

Mertens, F., Távora, R., Fonseca, I. F., Grando, R., Castro, M., \& Demeda, K. (2011). Redes sociais, capital social e governança ambiental no Território Portal da Amazônia. Acta Amazônica, 41(4), 481-492.

Novelli, M., Schmitz, B., \& Spencer, T. (2006). Networks, Clusters and Innovation in Tourism: a UK Experience. Tourism Management, 27(6), 1141-1152.

Okazaki, E. (2008). A Community-Based Tourism Model: It's Conception and Use. Journal of Sustainable Tourism, 16(5), 511-529.

Pforr, C. (2006). Tourism policy in the making: An Australian network study. Annals of Tourism Research, 33(1), 87-108.

Presenza, A., \& Cipollina, M. (2010). Analysing tourism stakeholders networks. Tourism Review, 65(4), 17-30.

Pretty, J. (2003). Social capital and the collective management of resources. Science, 302, 1912-1914.

Ramirez-Sanchez, S. (2011). The role of individual attributes in the practice of information sharing among fishers from Loreto, BCS, Mexico. In Ö. Bodin \& C. Prell (eds.), Social networks and natural resource management: uncovering the social fabric of environmental governance (pp. 234-254). Cambridge, UK: Cambridge University Press.

Rathwell, K. J., \& Peterson, G. D. (2012). Connecting Social Networks with Ecosystem Services for Watershed Governance: a Social-Ecological Network Perspective Highlights the Critical Role of Bridging Organizations. Ecology and Society, 17(2), 24.

Ruiz, E., Hernández, M., Coca, A., Cantero, P., \& Campo, A. (2008). Turismo comunitario en Ecuador: comprendiendo el community-based tourism desde la comunidad. Pasos, 6(3): 399-419.

Schärer, R. (2003). Turismo sustentável: um estudo de caso sobre a experiência da comunidade da Prainha do Canto Verde no litoral do Ceará. Pasos, 1(2), 231-242.

Scott, J. (2000). Social Network Analysis. A Handbook. London, UK: Sage Publications.

Scott, N., Baggio, R., \& Cooper, C. (2008). Network Analysis and Tourism: From Theory to Practice. Clevedon: Channel View Publications.
Shih, H. Y. (2006). Network characteristics of drive tourism destinations: An application of network analysis in tourism. Tourism Management, 27, 1029-1039

Swarbrooke, J. (2000). Turismo Sustentável: conceitos e impacto ambiental. São Paulo: Aleph.

Wasserman, S., \& Faust, K. (1999). Social Network Analysis: Methods and Applications. Cambridge: Cambridge University Press.

Webb, C. \& Bodin, Ö. (2008). A network perspective on modularity and control of flow in robust systems. In J. Norberg, \& G. Cumming (eds.), $A$ Theoretical Framework for Analyzing Social-Ecological Systems (pp. 85118). New York City: Columbia Univ Pr.

Woolcock, M., \& Narayan, D. (2000). Social capital: implications for development theory, research and policy. World Bank Research Observer 15(2), 225-249.

Recebido: 24.05.2016

Reenviado: 03.07.2016

Aceite: 04.07.2016 